SCIREA Journal of Mathematics http://www.scirea.org/journal/Mathematics

November 15, 2021

SCIREA

Volume 6, Issue 6, December 2021

\title{
A Note on Connected Six Cyclic Graphs Having Minimum Degree Distance
}

Nadia Khan, Fatima Ramazan and Munazza Shamas

Department of Mathematics and Statistics, The University of Lahore, Lahore Pakistan.

E-mail: nadiakhan811@gmail.com, fatimaramazan19@gmail.com, munazashms123@gmail.com

Mathematics Subject Classification: $05 \mathrm{C} 12$

\begin{abstract}
Let $G_{n}^{6}$ represents the class connected 6-cyclic graphs. In this paper, first some result is derived for the characterization of class connected 6-cyclic graphs. Then we find minimum degree distance of class of connected of 6-cyclic graph.
\end{abstract}

Keywords: connected graph, degree distance, Six cyclic graphs

\section{Introduction}

For any graph $G \in G_{n}, d(x, y)$ represents the shortest distance between the vertices $x, y \in$ $V(G)$ and the maximum of $d(x, y)$ for any vertices $x, y \in V(G)$ is defined to be the diameter of $G$, denoted by $\operatorname{giam}(G)$. 
The Wiener index [8] is a well-known topological index of a molecular graph which is used to describe molecular branching and cyclicity. It's also stablishes correlation with various parameters of chemical compounds. The wiener index of a graph $G$ is defined as

$$
\sum_{x \in V(G)} \sum_{x \in V(G)} d(x, y)
$$

Dobrynin and Kotchetova [1] and Gutman [2] introduced a new graph invariant that is more sensitive than the Wiener index. It is defined in the following way: given any $G \in G_{n}$ the degree distance of a vertex $x \in V(G)$ is defined by

$$
D^{\prime}(x)=d(x) D(x)
$$

where $d(x)$ is the degree of $x$ and $D(x)=\sum_{x, y \in V(G)} d(x, y)$. The degree distance of a graph $G$ is defined as:

$$
D^{\prime}(G)=\sum_{x \in V(G)} D^{\prime}(x)=\sum_{x \in V(G)} d(x) D(x)=\frac{1}{2} \sum_{x, y \in V(G)} d(x, y)(d(x)=d(y)) .
$$

In this paper, we determine all the extremal 5-cyclic graphs achieving the minimum degree distance.

In section 2, we list some known results needed in this paper. In section 3, we characterize extremal 5-cyclic graphs achieving the minimum degree distance.

\section{Some Lemmas}

In this section, we characterize connected six cyclic graphs by their degree sequence in the following lemma.

Lemma 1. Let $n \geq 6$. The degrees of the vertices of a graph $G \in G_{n}^{6}$ are the integers $n-1 \geq$ $d_{1} \geq d_{2} \geq \cdots \geq d_{n} \geq 1$, iff

(i) $\sum_{i=1}^{n} d_{i}=2 n+10$

(ii) $d_{i} \geq 2$, for at least six indices.

Proof: $\Rightarrow$ Let $G \in G_{n}^{5}$. Then by the definition of 5-cyclic graph condition (i) and (ii) is verified. 
$\Leftarrow$ For $n=6$, we have $\sum_{i=1}^{6} d_{i}=22$. If $d_{6} \geq 4$ then $d_{1}+d_{2}+d_{3}+d_{4}+d_{5} \geq 24$ a contradiction. So $d_{6}<4$. If $d_{6}=3$ then $\sum_{i=1}^{n} d_{i}=19$, which implies that $\left(d_{1}, d_{2}, d_{3}, d_{4}, d_{5}, d_{6}\right)=(5,5,3,3,3,3)$, or $(5,4,4,3,3,3)$ or $(4,4,4,4,3,3)$ represented by graphs $H_{1}$ or $H_{2}$ or $H_{3}$, respectively in fig 1 . If $d_{6}<3$, then $d_{6}=2$ and $\left(d_{1}, d_{2}, d_{3}, d_{4}, d_{5}, d_{6}\right)=(5,4,4,4,3,2)$, or $(5,5,4,3,3,2)$ represented by graphs $H_{4}$ or $H_{5}$, respectively in fig 1 . Let $n \geq 7$ and assume that the result is true for all $k \leq n$.

Case 1. If $d_{n}>1$, then $d_{n}=2$. Otherwise, $d_{1}+d_{2}+\cdots+d_{n}>3 n>2 n+10$. If $d_{n}=3$ for $\mathrm{n}=7,8,9$ or 10 , we do not have a graphical sequence. For $d_{n}=2$, and sufficiently large $n$, by solving the equation, $d_{1}+d_{2}+\cdots+d_{n-1}=2 n+8$. We have the following possibilities

Subcase 1.1. If $d_{1}=12, d_{2}=d_{3}=\cdots=d_{n}=2$, then the unique resultant graph is isomorphic to $G_{1}$ in fig. 2 .

Subcase 1.2. If $d_{1}=11, d_{2}=3, d_{3}=\cdots=d_{n}=2$, then the unique resultant graph is isomorphic to $G_{2}$ in fig. 2 .

Subcase 1.3. If $d_{1}=10, d_{2}=d_{3}=3, d_{4}=\cdots=d_{n}=2$, then the unique resultant graph is isomorphic to $G_{3}$ or $G_{4}$ in fig.2.

Subcase 1.4. If $d_{1}=9, d_{2}=d_{3}=d_{4}=4, d_{5}=d_{6}=\cdots=d_{n}=2$, then the unique resultant graph is isomorphic to $G_{5}$ or $G_{6}$ in fig.2.

Subcase 1.5. If $d_{1}=8, d_{2}=d_{3}=d_{4}=d_{5}=3, d_{6}=\cdots=d_{n}=2$, then the unique resultant graph is isomorphic to $G_{7}$ or $G_{8}$ in fig.2.

Subcase 1.6. If $d_{1}=7, d_{2}=4, d_{3}=d_{4}=d_{5}=3, d_{6}=\cdots=d_{n}=2$, then the unique resultant graph is isomorphic to $G_{9}$ in fig. 2 .

Subcase 1.7. If $d_{1}=6, d_{2}=d_{3}=4, d_{4}=d_{5}=3, d_{6}=\cdots=d_{n}=2$, then the unique resultant graph is isomorphic to $G_{10}$ or $G_{11}$ in fig.2.

Subcase 1.8. If $d_{1}=6, d_{2}=4, d_{3}=d_{4}=d_{5}=d_{6}=3, d_{7}=\cdots=d_{n}=2$, then the unique resultant graph is isomorphic to $G_{12}$ in fig.2.

Subcase 1.9. If $d_{1}=d_{2}=6, d_{3}=d_{4}=4, d_{5}=d_{6}=\cdots=d_{n}=2$, then the unique resultant graph is isomorphic to $G_{13}$ in fig.2.

Subcase 1.10. If $d_{1}=6, d_{2}=5, d_{3}=4, d_{4}=3, d_{5}=d_{6} \cdots=d_{n}=2$, then the unique resultant graph is isomorphic to $G_{14}$ in fig.2. 
Subcase 1.11. If $d_{1}=6, d_{2}=d_{3}=d_{4}=d_{5}=d_{6}=d_{7}=3, d_{8}=\cdots=d_{n}=2$, then the unique resultant graph is isomorphic to $G_{15}$ in fig.2.

Subcase 1.12. If $d_{1}=5, d_{2}=d_{3}=d_{4}=d_{5}=d_{6}=d_{7}=3, d_{8}=\cdots=d_{n}=2$, then the unique resultant graph is isomorphic to $G_{16}$ in fig. 2 .

Subcase 1.13. If $d_{1}=d_{2}=5, d_{3}=d_{4}=4, d_{5}=d_{6}=3, d_{7}=\cdots=d_{n}=2$, then the unique resultant graph is isomorphic to $G_{17}$ in fig.2.

Subcase 1.14. If $d_{1}=d_{2}=5, d_{3}=4, d_{4}=d_{5}=3, d_{6}=d_{7}=d_{8}=\cdots=d_{n}=2$, then the unique resultant graph is isomorphic to $G_{18}$ in fig. 2 .

Subcase 1.15. If $d_{1}=d_{2}=d_{3}=d_{4}=d_{5}=4, d_{6}=d_{7}=\cdots=d_{n}=2$, then the unique resultant graph is isomorphic to $G_{19}$ in fig. 2 .

Subcase 1.16. If $d_{1}=d_{2}=d_{3}=d_{4}=d_{5}=d_{6}=d_{7}=d_{8}=d_{9}=d_{10}=3, d_{11}=\cdots=$ $d_{n}=2$, then the unique resultant graph is isomorphic to $G_{20}$ in fig.2.

Subcase 1.17. If $d_{1}=5, d_{2}=4, d_{3}=d_{4}=d_{5}=d_{6}=d_{7}=3, d_{8}=\cdots=d_{n}=2$, then the unique resultant graph is isomorphic to $G_{21}$ in fig.2.

Case 2. If $d_{n}=1$, we consider the following two subcases:

Subcase 2.1. If $d_{1}=n-1$, and for sufficiently large $n$, we have the following possibilities,

Subcase 2.1.1. If $d_{1}=n-1, d_{2}=d_{3}=d_{4}=3, d_{5}=\cdots=d_{10}=2, d_{11}=\cdots=d_{n}=1$, then the unique resultant graph is isomorphic to $A_{1}$ in fig.3.

Subcase 2.1.2. If $d_{1}=n-1, d_{2}=\cdots=d_{7}=3, d_{11}=\cdots=d_{n}=1$, then the unique resultant graph is isomorphic to $A_{2}$ in fig.3.

Subcase 2.1.3. If $d_{1}=n-1, d_{2}=5, d_{3}=3, d_{4}=d_{5}=d_{6}=d_{7}=2, d_{8}=\cdots=d_{n}=1$, then the unique resultant graph is isomorphic to $A_{3}$ in fig.3.

Subcase 2.1.4. If $d_{1}=n-1, d_{2}=5, d_{3}=3, d_{4}=d_{5}=d_{6}=3, d_{7}=d_{8}=2, d_{9}=\cdots=$ $d_{n}=1$, then the unique resultant graph is isomorphic to $A_{4}$ in fig.3.

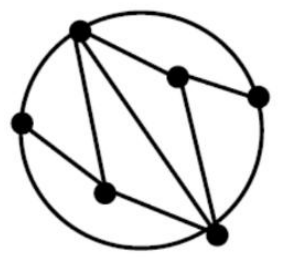

$\mathrm{H}_{1}$

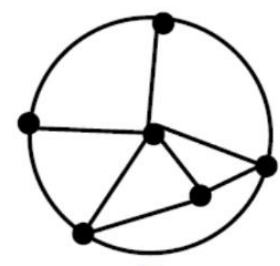

$\mathrm{H}_{2}$

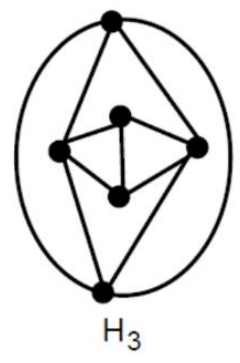

$\mathrm{H}_{3}$

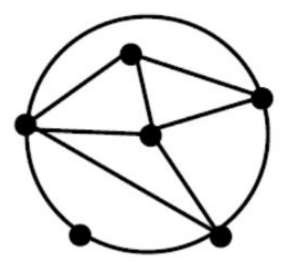

$\mathrm{H}_{4}$

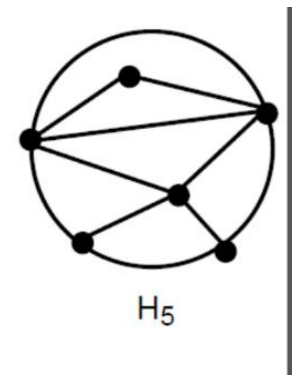


Fig. 1

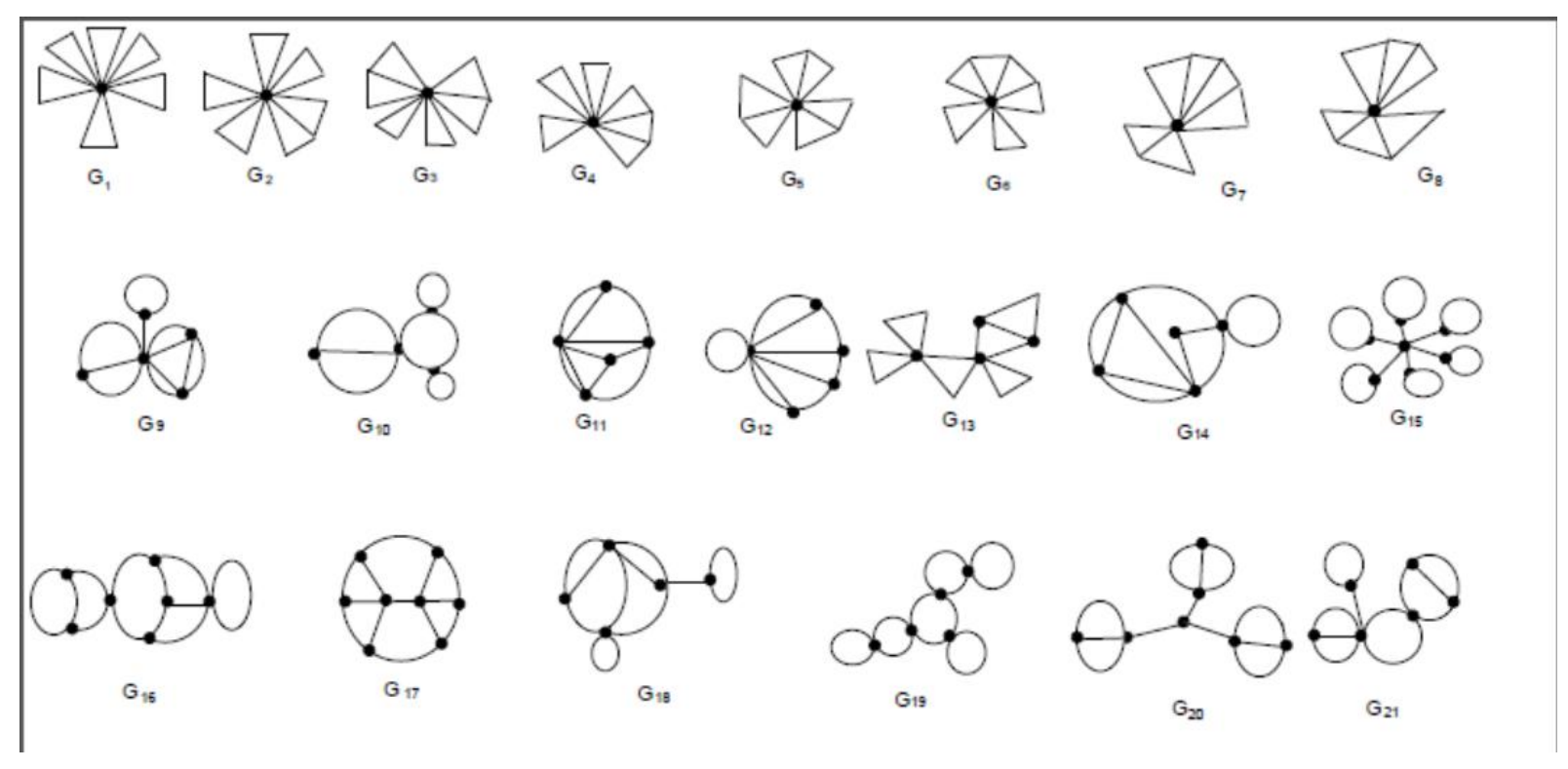

Fig. 2

Subcase 2.1.5. If $d_{1}=n-1, d_{2}=\cdots=d_{5}=3, d_{6}=\cdots=d_{9}=2, d_{10}=\cdots=d_{n}=1$, then the unique resultant graph is isomorphic to $A_{5}$ in fig.3.

Subcase 2.1.6. If $d_{1}=n-1, d_{2}=4, d_{3}=d_{4}=d_{5}=3, d_{6}=d_{7}=2, d_{8}=d_{9}=\cdots=$ $d_{n}=1$, then the unique resultant graph is isomorphic to $A_{6}$ in fig.3.

Subcase 2.1.7. If $d_{1}=n-1, d_{2}=d_{3}=d_{4}=d_{5}=d_{6}=d_{7}=3, d_{8}=\cdots d_{19}=2, d_{20}=$ $\cdots=d_{n}=1$, then the unique resultant graph is isomorphic to $A$ in fig.3.

Subcase 2.1.8. If $d_{1}=n-1, d_{2}=7, d_{3}=d_{4}=d_{5}=d_{6}=d_{7}=d_{8}=2, d_{9} \cdots=d_{n}=1$, then the unique resultant graph is isomorphic to $A$ in fig.3.

Subcase 2.1.9. If $d_{1}=n-1, d_{2}=4, d_{3}=d_{4}=3, d_{5}=d_{6}=d_{7}=d_{8}=2, d_{9}=\cdots=$ $d_{n}=1$, then the unique resultant graph is isomorphic to $A_{9}$ in fig.3.

Subcase 2.1.9. If $d_{1}=n-1, d_{2}=d_{3}=4, d_{4}=3, d_{5}=d_{6}=d_{7}=2, d_{8}=\cdots=d_{n}=1$, then the unique resultant graph is isomorphic to $A_{10}$ in fig.3. 


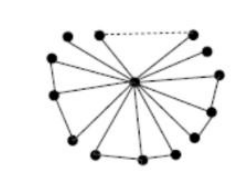

$A_{1}$
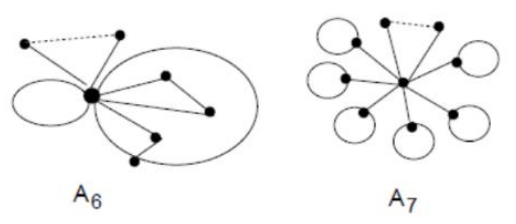

$\mathrm{A}_{7}$

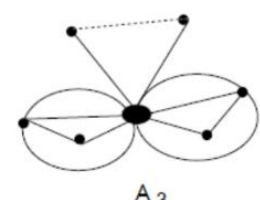

$\mathrm{A}_{3}$
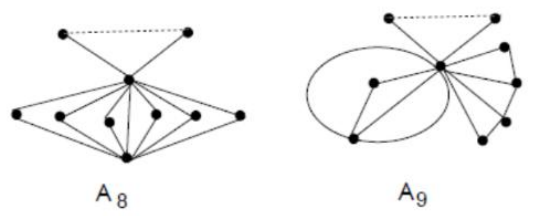

$\mathrm{A}_{9}$
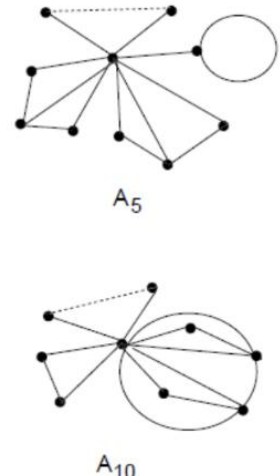

Fig 3.

Subcase 2.2. Suppose $d_{1} \leq n-2$ and $d_{n}=1$. For each $1 \leq i \leq n-1$ if $d_{i} \leq 2$ then $\sum_{i} d_{i} \leq 2 n-1$, which is a contradiction. We can find a maximal index $j, 1 \leq j \leq n-1$, such that $d_{j} \geq 3$, and $d_{j+1} \leq 2$ and $d_{1} \geq d_{2} \geq \cdots \geq d_{j-1} \geq d_{j} \geq \cdots \geq d_{n} \geq 1$. At least six members of the sequence $d_{1}, \cdots, d_{j-1}, d_{j}-1, \cdots, d_{n-1}$ are greater than 2 , for which $d_{1} \leq$ $n-2$ and $d_{1}+d_{2}+\cdots+d_{n}=2(n-1)+10=2 n+8$. By induction hypothesis there exist $G \in G_{n}^{6}$ having this degree sequence. By adding a new vertex, joined by an edge with the vertex having degree $d_{j-1}$, we obtain a graph having six cycles with the degree sequence $d_{1} \geq d_{2} \geq \cdots \geq d_{n}=1$. This completes the proof of Lemma 1 .

If for any vertex $v \in V(G), d(v)=k$, then $D(v) \geq 2 n-k-2$, and if for all $v \in V(G), \quad d(v, y) \leq 2$ then $D(v)=2 n-k-2 \quad$. Consequently, $D^{\prime}(G)=$ $\sum_{v \in V(G)} d(v) D(v) \geq \frac{1}{2} \sum_{k=1}^{n-1} k x_{k}(2 n-k-2)$, where $x_{i}$ denotes the number of vertices of degree $i, 1 \leq i \leq n-1$. By denoting as in [2], $F\left(x_{1}, x_{2}, \cdots, x_{n-1}\right)=\sum_{k=1}^{n-1} k x_{k}(2 n-k-2)$. We will find the minimum of $F\left(x_{1}, x_{2}, \cdots, x_{n-1}\right)$ over all-natural numbers $x_{1}, x_{2}, \cdots, x_{n-1} \geq$ 0 satisfying the conditions in above lemma. We have the following corollary:

Corollary: Let $n \geq 6$. The integers $x_{1}, x_{2}, \cdots, x_{n-1} \geq 0$ are the multiplicities of the degrees of a graph $G \in G_{n}^{6}$ iff

(i) $\sum_{i=1}^{n-1} x_{i}=n$

(ii) $\sum_{i=1}^{n-1} i x_{i}=2 n+10$

(iii) $x_{1} \leq n-6$ 
Let the set of vectors $x_{1}, x_{2}, \cdots, x_{n-1}$, where $x_{1}, \cdots, x_{n-1}$ are non-negative integers be denoted by $\Delta$ satisfying the conditions (i)-(iii) of corollary. Let us define transformations $T_{1}$ and $T_{2}$ $m \geq 2, p>0, m+p \leq n-2, x_{m} \geq 1, x_{p} \geq 1$, by

$T_{1}\left(x_{1}, \cdots, x_{n-1}\right)=\left(x_{1}^{\prime}, \cdots, x_{n-1}^{\prime}\right)=\left(x_{1}, \cdots, x_{m-1}+1, x_{m}-1, \cdots, x_{m+p}-1, x_{m+p+1}+\right.$ $\left.1, \cdots, x_{n-1}\right)$ and

$T_{2}\left(x_{1}, \cdots, x_{n-1}\right)=\left(x_{1}^{\prime}, \cdots, x_{n-1}^{\prime}\right)=\left(x_{1}, \cdots, x_{m-1}+1, x_{m}-2, x_{m+1}+1, \cdots, x_{n-1}\right)$ we have $x_{i}=x_{i}^{\prime}$ for $i \neq\{\mathrm{m}-1, \mathrm{~m}, \mathrm{~m}+\mathrm{p}, \mathrm{m}+\mathrm{p}+1\}$.

Lemma 2. Let $\left(x_{1}, \cdots, x_{n-1}\right) \in \Delta$ then

(a). $T_{1}\left(x_{1}, \cdots, x_{n-1}\right) \in \Delta$ if $m \neq 2$ and $x_{1} \neq n-7$, moreover $F\left(T_{1}\left(x_{1}, \cdots, x_{n-1}\right)\right)<$ $F\left(x_{1}, \cdots, x_{n-1}\right)$

(b). $T_{2}\left(x_{1}, \cdots, x_{n-1}\right) \in \Delta$ if $m \neq 2$ and $x_{1} \neq n-7$, moreover $F\left(T_{2}\left(x_{1}, \cdots, x_{n-1}\right)\right)<$ $F\left(x_{1}, \cdots, x_{n-1}\right)$

Proof: (a). As $\sum_{i=1}^{n-1} x_{i}=\sum_{i=1}^{n-1} x_{i}^{\prime}$ and $\sum_{i=1}^{n-1} i x_{i}=\sum_{i=1}^{n-1} i x_{i}^{\prime}=2 n+6$. If $\left(x_{1}, \cdots, x_{n-1}\right) \in \Delta$, $m=2$ and $n-6$ then $x_{1}^{\prime}>n-6$ a contradiction. Also $F\left(x_{1}, \cdots, x_{n-1}\right)-$ $F\left(T_{1}\left(x_{1}, \cdots, x_{n-1}\right)\right)=2 p+2>0$.

Similarly, (b) also hold. This completes the proof of lemma 2.

\section{Main result}

Theorem: Let $G \in G_{n}^{6}$,

(a). If $n=6$ then $\min D^{\prime}(G)=132$, and the unique extremal graph is isomorphic to $H_{2}$ as shown in fig.1.

(b). If $n \geq 7$ then $\min ^{\prime}(G)=3 n^{2}+17 n-80$ then all the extremal graphs are isomorphic to the graphs $F_{1}$ and $F_{2}$ in fig.5.

Proof. In order to find $\min F\left(x_{1}, \cdots, x_{n-1}\right)$ where $\left(x_{1}, \cdots, x_{n-1}\right) \in \Delta$.

Firstly, let $n=6$ the resultant graphs are given in Fig 1 , and $D^{\prime}\left(H_{2}\right)=132$ is the minimum value.

Secondly, let us consider $n=7$. Then all graphs $G \in G_{7}^{6}$ are $C_{i}$ 's where $1 \leq i \leq 11$. Here 
$D^{\prime}\left(C_{1}\right)=D^{\prime}\left(C_{7}\right)=202, D^{\prime}\left(C_{2}\right)=190, D^{\prime}\left(C_{3}\right)=200, D^{\prime}\left(C_{4}\right)=D^{\prime}\left(C_{6}\right)=198, D^{\prime}\left(C_{5}\right)=$ 194, $D^{\prime}\left(C_{8}\right)=188, D^{\prime}\left(C_{9}\right)=D^{\prime}\left(C_{10}\right)=198$ and $D^{\prime}\left(C_{11}\right)=186$, where $C_{i}$ are shown in

Fig.4.

Finally, $n \geq 8$. If $x_{n-1} \geq 2$, consider two different vertices $u, v \in V(G)$ such that $d(u)=$ $d(v)=n-1$. As $n \geq 8$, we can choose at least eight different vertices distinct from $u, v$ which are adjacent to $u, v$. Thus, we have at least seven cycles, which contradicts the hypothesis, Therefore $x_{n-1} \leq 1$.

Now we analyze the possible values of $x_{1}, x_{2}, \cdots, x_{n-2}$ If there exist $8<i, j<n-2$ such that $x_{i} \geq 1$ and $x_{j} \geq 1$ then by applying the transformation $T_{1}$ for the position $i$ and $j$, we have a new vector $\left(x_{1}^{\prime}, \cdots, x_{n-1}^{\prime}\right) \in \Delta$ for which $\mathrm{F}\left(x_{1}^{\prime}, \cdots, x_{n-1}^{\prime}\right)<F\left(x_{1}, \cdots, x_{n-1}\right)$. Similarly, if there exist $6<i, j<n-2$ such that $x_{i} \geq 2$ then we obtain a new degree sequence in $\Delta$ for which $\mathrm{F}\left(x_{1}^{\prime}, \cdots, x_{n-1}^{\prime}\right)<F\left(x_{1}, \cdots, x_{n-1}\right)$. by applying the transformation $T_{2}$. Now we consider two cases:

Case 1. Suppose that there exist distinct indices $i$, $k$ with $8<i, k<n-2$ such that $x_{i}=1$ and $x_{k}=0$. In this case, if $x_{7} \geq 1$ then by applying the transformation $T_{1}$ for position 7 and $i$, we obtain smaller a smaller value of $F$, Suppose that $x_{7}=0$. Since $x_{n-1} \in\{0,1\}$, we will analyze the two cases separately.

(a) In this case $x_{n-1}=x_{i}=1$, where $7<i$ and $x_{5}=0$. We can consider different vertices $\mathrm{u}, \mathrm{v}, \mathrm{w}, \mathrm{x}, \mathrm{y}, \mathrm{p}, \mathrm{q} \in \mathrm{V}(\mathrm{G})$ such that $\mathrm{d}(\mathrm{u})=\mathrm{n}-1, \mathrm{~d}(\mathrm{v})=\mathrm{i} \geq 7$ then $\mathrm{w}, \mathrm{x}, \mathrm{y}, \mathrm{p}, \mathrm{q}$ are all adjacent to $\mathrm{u}$ and $\mathrm{v}$ respectively. Meanwhile, $\mathrm{u}$ and $\mathrm{v}$ are adjacent too, so we have found six cycles which contradicts the hypothesis.

(b) If $x_{n-1}=0$ then $x_{5}=0$ and $x_{i}=1,(7<i, n-2)$ and $\Delta$ is characterized by the equations $x_{1}+x_{2}+x_{3}+x_{4}=n-1$ and $x_{1}+2 x_{2}+3 x_{3}+4 x_{4}=2 n+8-i$ which implies that $x_{2}+2 x_{3}=n+8-i$ by solving for $x_{2}$ and $x_{3}$ and then by applying the transformation for position 2 and $i$ or 3 and $i$ or 4 and $i$, we obtain smaller value of $F$.

Case 2. Suppose that $x_{8}, \cdots, x_{n-1}=0$, hold and the degree sequence is $\left(x_{1}, x_{2}, x_{3}, x_{4}, x_{5}, x_{6}, x_{7}, 0, \cdots, 0, x_{n}\right)$. As $x_{n-1} \in\{0,1\}$, so we have to analyze two cases:

(a'). If $x_{n-1}=0$, then $x_{2}+2 x_{3}+3 x_{4}+4 x_{5}+5 x_{6}+6 x_{7}=n+10$. This equation does not hold. If all $x_{2}, x_{3}, x_{4}, x_{5}, x_{6}$ and $x_{7}$ are not greater than 2 , then $x_{2}+2 x_{3}+3 x_{4}+4 x_{5}+$ $5 x_{6}+6 x_{7} \leq 42$ which contradicts the hypothesis $n \geq 8$. If one of them is greater than two, then by using $T_{2}$ for the corresponding position, we obtain a smaller value of $F$. 
(b'). If $x_{n-1}=1$, then $x_{2}+2 x_{3}+3 x_{4}+4 x_{5}+5 x_{6}+6 x_{7}=12$. If $x_{7} \geq 3$, then $x_{2}+$ $2 x_{3}+3 x_{4}+4 x_{5}+5 x_{6}+6 x_{7} \geq 18$ which is not possible. So $x_{7} \leq 2$, if $x_{7}=2$ then $x_{2}+$ $2 x_{3}+3 x_{4}+4 x_{5}+5 x_{6}=0$, which implies that $x_{2}=x_{3}=x_{4}=x_{5}=x_{6}=0$ and $x_{1}=n-$ 3 which is a contradiction as $x_{1} \leq n-6$. So $x_{7} \neq 2$. Thus either $x_{7}=0$ or $x_{7}=1$.

If $x_{7}=1$, then $x_{2}+2 x_{3}+3 x_{4}+4 x_{5}+5 x_{6}=6$, the possible solutions which gives the graphical degree sequence and which satisfy all the conditions of corollary 1 is $x_{2}=6, x_{3}=$ $x_{4}=x_{5}=x_{6}=0$. The degree sequence $(\mathrm{n}-8,6,0,0,0,0,1,0, \cdots, 0,1)$ and $t_{2}(\mathrm{n}-$ $8,6,0,0,0,0,1,0, \cdots, 0,1)$ is not graphical. Thus $F(\mathrm{n}-8,6,0,0,0,0,1,0, \cdots, 0,1)=3 n^{2}+$ $17 n-86$.

Next consider if $x_{7}=0$, then then $x_{2}+2 x_{3}+3 x_{4}+4 x_{5}+5 x_{6}=12$, all possible solution of $x_{2}, x_{3}, x_{4}, x_{5}$ and $x_{6}$ which follows the above corollary and construct the twenty dour degree sequences which are graphical. The sequences are

$(\mathrm{n}-6,0,3,2,0, \cdots, 0,1),(\mathrm{n}-6,0,4,0,1,0, \cdots, 0,1),(n-7,3,0,3,0, \cdots, 0,1)$,

$(n-7,0,6,0, \cdots, 0,1),(\mathrm{n}-7,2,3,0,1,0, \cdots, 0,1),(\mathrm{n}-7,1,4,1,0, \cdots, 0,1),(\mathrm{n}-7,3,1,1,1, \cdots, 0,1)$, $(n-8,4,1,2,0, \cdots, 0,1),(n-8,3,3,1,0, \cdots, 0,1),(n-8,2,5,0, \cdots, 0,1),(n-8,5,1,0,0,1,0, \cdots, 0,1)$, $(n-7,3,2,0,0,1,0, \cdots, 0,1),(\mathrm{n}-9,5,2,1,0, \cdots, 0,1),(\mathrm{n}-9,6,1,0,1,0, \cdots, 0,1),(\mathrm{n}-9,6,0,2,0 \cdots, 0,1)$, $(n-9,7,0,0,0,1, \cdots, 0,1),(\mathrm{n}-10,8,0,0,1,0, \cdots, 0,1),(\mathrm{n}-10,7,1,1,0, \cdots, 0,1),(\mathrm{n}-10,6,3,0, \cdots, 0,1)$, $(n-11,8,2,0, \cdots, 0,1),(n-11,9,0,1,0, \cdots, 0,1),(n-12,10,1,0, \cdots, 0,1),(n-13,12,0, \cdots, 0,1)$.

By applying transformations $T_{1}$ and $T_{2}$ these degree sequences are transformed to either the sequence $(\mathrm{n}-7,3,2,0,0,1,0, \cdots, 0,1)$ or $(\mathrm{n}-6,1,2,1,1,0, \cdots, 0,1)$ represented by graphs $F_{1}$ and $F_{2}$ respectively. But $F(\mathrm{n}-7,3,2,0,0,1,0, \cdots, 0,1)=3 n^{2}+17 n-80<F(\mathrm{n}-$ $6,1,2,1,1,0, \cdots, 0,1)=3 n^{2}+17 n-78$. Hence for $n \geq 7, \min ^{\prime}(G)=3 n^{2}+17 n-80$.

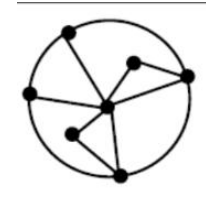

$\mathrm{C}_{1}$

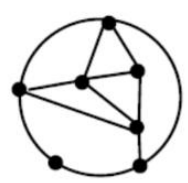

$\mathrm{C}_{7}$

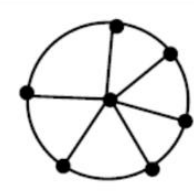

$\mathrm{C}_{2}$

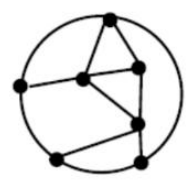

$\mathrm{C}_{8}$
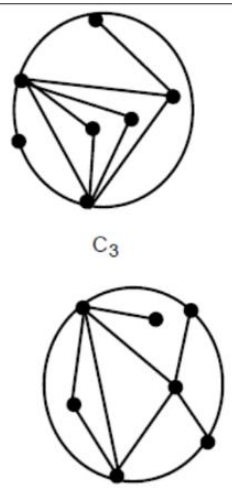

$\mathrm{C}_{9}$
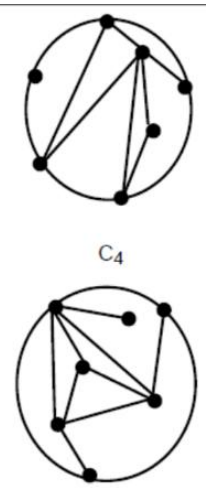

$\mathrm{C}_{10}$
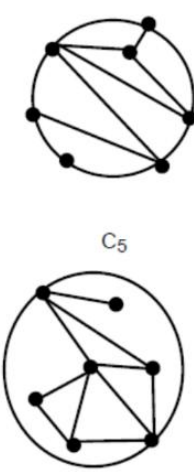

$\mathrm{C}_{11}$

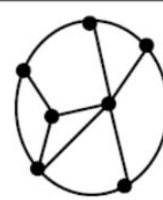

$\mathrm{C}_{6}$

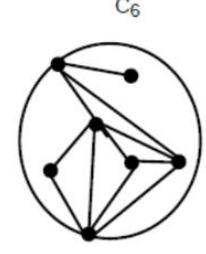

$\mathrm{C}_{12}$

Fig. 4 

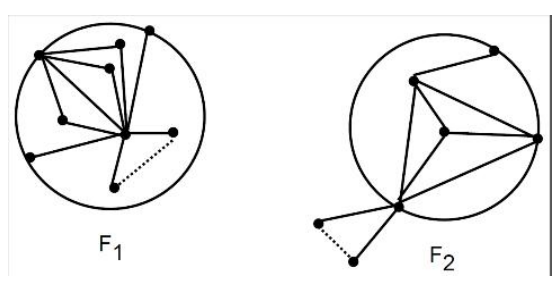

Fig. 5

\section{References}

[1] A. A. Dobrynin and A. A. Kochetova, A Degree distance of a graph: A degree analogue of the Wiener index, J. Chem. Inform. Comput. Sci., 34(1994), 1082-1086.

[2] I. Gutman, Selected properties of the Schultz molecular topological index, J. Chem. Inform. Comput. Sci\}, 34(1994), 1087-1089.

[3] J. W. Moon, Counting Labelled Trees, Canadian Mathematical Monographs\}, Vol. 1, W. Clowes and Sons, London and Beccles, (1970).

[4] J. K. Senior, Partitions and their representative graphs, Amer. J. Math., 73(1951), 663689.

[5] I. Tomescu, Some extremal properties of the degree distance of a graph, Discrete Appl. Math., 98 (1999), 159-163.

[6] A. I. Tomescu, Note on unicyclic and bicyclic graphs having minimum degree distance, Discrete Appl. Math., 156(2008), 125-130.

[7] W. Zhu, A note on tricyclic graphs with minimum degree distance, Discrete. Math. Algorithms and applications., Vol 3, No. 1 (2011) 25-32.

[8] H. Hosoya, Topological index. A newly proposed quantity characterizing the topological nature of structural isomers of saturated hydrocarbons, Bull. Chem. Soc Jpn., 4(1971), 2332-2339. 